International Journal of Engineering \& Technology, 7 (4.6) (2018) 9-12
International Journal of Engineering \& Technology
SPC
Website: www.sciencepubco.com/index.php/IJET
Research paper

\title{
Human Emotion Surveillance Using Computer Vision
}

\author{
Dr.R.Radha ${ }^{1}$, Atchatha.M ${ }^{2}$, Kaushik.B ${ }^{3}$, Agassi Felix $A^{4}$ \& G. Staflin Betzy ${ }^{5}$ \\ ${ }^{1}$ Department of Computer Science and Engineering, KL University, Hyderabad, India \\ ${ }^{2}$ Department of Information Technology, Easwari Engineering College, Chennai, India \\ ${ }^{3}$ Department of Information Technology, Easwari Engineering College, Chennai, India \\ ${ }^{4}$ Department of Information Technology, Easwari Engineering College, Chennai, India \\ ${ }^{5}$ Department of Information Technology, Easwari Engineering College, Chennai, India \\ *Corresponding author E-mail: radhupriya@yahoo.co.in
}

\begin{abstract}
India, a land of marvels, is outstanding in many aspects, its culture, ecosystem, etc. Sadly, it also ranks among the top countries in the world to have an annual suicide rate. This project aims at the foundation of human emotion surveillance. This system assists in the facial recognition, feature extraction and the threshold detection of stress for emotions expressed through face using the viola-jones algorithms and weak classifiers. This focuses basically on segregation of positive and negative emotions, detecting stress based on a u sual threshold value and possibly providing an alternate means to let loose the extra stress built up if possible.
\end{abstract}

Keywords: Facial recognition, viola-jones algorithm, weak classifier.

\section{Introduction}

India, a country filled with culture and rich heritage portraits itself as one of the most leading countries in many fields, but sadly it also ranks one of the tops in the rates of suicides attempted per year. According to a WHO report published in Thursday which found that one person commits suicide every 40 seconds globally. India ranked in the tops in suicide rates in 2012 of which most are in the age group of 15-29 years of age ${ }^{[1]}$.

\section{Suicides in India - 2014}

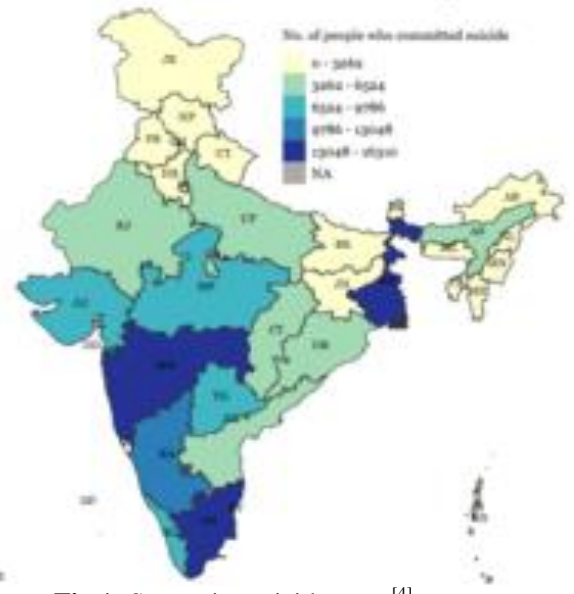

Fig.1: State wise suicide rates ${ }^{[4]}$

Every year about 30 to 44 people per 100,000 Indians aged between 15 and 29 kill themselves ${ }^{[2]}$. Of the 134799 suicides in the year $2013,32325^{[3]}$ deaths were from family issues, a quarter of the whole, which could have been resolved with a proper sitting. Of the deaths in the age group of 15 and 29, most are because of the parental pressure to fare well in the board examinations ${ }^{[3][4]}$.

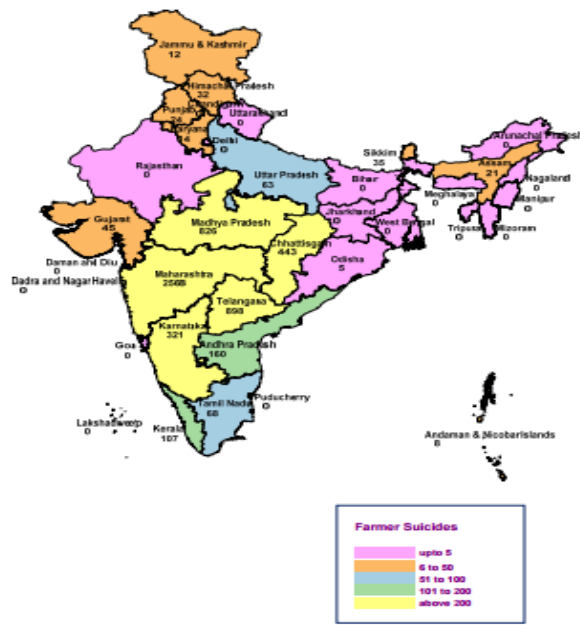

Fig.2: State wise farmer suicide rates ${ }^{[6]}$

15 people commits suicide every day in India, with statistics showing Tamil Nadu as having the most deaths in $2014^{[5]}$. Farmer suicides in the country rose by $42 \%$ between 2014 and 2015, according to newly released data from the National Crime Records Bureau (NCRB). It recorded 5,650 suicides by farmers and cultivators in 2014. The figure rose to 8,007 in the latest data ${ }^{[5]}$. The Major reasons for these attempts are low self-esteem, loneliness, lack of care etc., these are the factors that take over the emotions over a time period and causes depression making them take the worst decision - suicide. Our project aims at deducing the stress of the person before it reaches to that unfavorable outcome.

The paper is divided into five sections. Section I deals with the introduction. Section II gives a glimpse of the pre-existing techniques. Section III gives a detailed overview of the methodology. 
Section IV states the results obtained. Section V has the conclusion and the future indications.

\begin{tabular}{|c|c|c|}
\hline \multicolumn{3}{|c|}{ States with highest number of farmer suicides in 2015} \\
\hline State & No. of suicides & Share of total (\%) \\
\hline Maharashtra & 3,030 & 37.8 \\
\hline Telangana & 1,358 & 17 \\
\hline Karnataka & 1,197 & 14.9 \\
\hline Chhattisgarh & 854 & 10.7 \\
\hline Madhya Pradesh & 581 & 7.3 \\
\hline Andhra Pradesh & 516 & 6.4 \\
\hline
\end{tabular}

Fig.3: States with highest suicides in $2015^{[5]}$

\section{Literature Survey}

\subsection{Face detection}

Various methods have been developed for detecting the face. This has undergone a long and wide journey ranging from the detection based on color threshold, crust-trough method to many sophisticated feature based algorithms like viola-jones and Kanade-lucas Tomasi (KLT) algorithm. The difference in the color of the face from the rest of the background was utilized in color thresholding; this was a poor method for the purpose as during the presence of any light-colored wall (background) or some peach colored shirt can be misinterpreted as the skin, leading to miscalculations. Also, there are variations to the skin color. The surface near the mouth and the eyes form a trough in the processed image of the face, this also proved to be not as effective owing to the fact that the image may contain many other regions with troughs. Thus, we move onto the feature based advanced methods. The Viola-Jones object detection framework $^{[6]}$, proposed in 2001 by Paul Viola and Michael Jones, is the first framework to provide competitive object detection rates in real-time. It was motivated mainly by the problem of face detection. This face detection framework is capable of processing images extremely rapidly while achieving high detection rates.

\subsection{Emotion detectors}

Early works are based on either static images or for specific customized faces. Damir et.al ${ }^{[7]}$ had proposed a system that classifies based on feed tum data. Neha et.al ${ }^{[8]}$ had proposed a system based on color and feature based system. Both the above systems work only on static images. Seyedehsamaneh et.al ${ }^{[9]}$ had proposed a system wherein the images are detected using optical flow method with Extreme Sparse Learning. This in our case of detecting only the negative emotions seems to be complex.

Pre-existing systems like AFFECTIVA ${ }^{[10]}$ are a few proprietary software that help detect the human emotional response. AFFECTIVA is an emotion measurement technology company that grew out of MIT's Media Lab, which has developed a way for computers to recognize human emotions based on facial cues or physiological responses. Among the commercial applications, this emotion recognition technology is used to help brands improve their advertising and marketing messages. Another major application has been in political polling. Hence, here is our project aiming at the detection of various emotions of a human through the facial feature extraction.

\subsection{Other methods of detection}

Apart from face there are other methods for detecting the emotional level of the individual. Speech recognition ${ }^{[11]}$, web mining of suicide blogs ${ }^{[12]}$, observation of EEG signals ${ }^{[13]}$ are a few alternative methods. But these cannot be used as a primary means of detection as they are not as reliable. These can be augmented to the facial emotion detection technique to improve the recognition.

\section{Methodology}

Face speaks more than the words. Face of the subject is captured using the camera module. The detected image is processed to identify the face of the subject using viola-jones algorithm. This detected face is processed and the emotions are classified as either positive or negative emotions. This is plotted and an increase in the negative emotion can be inferred as increase in stress.

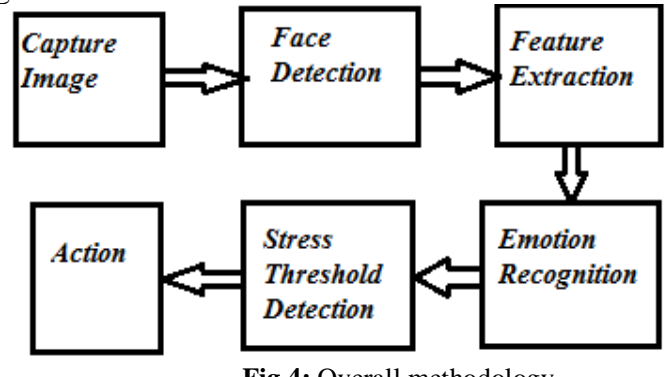

Fig.4: Overall methodology

\subsection{Face detection}

The image captured using the camera module is processed using viola-jones algorithm which uses Haar like features to get the bounding box around the face. Haar features are geometric features that assist in identifying the features that are geometric in nature and have some difference in color.
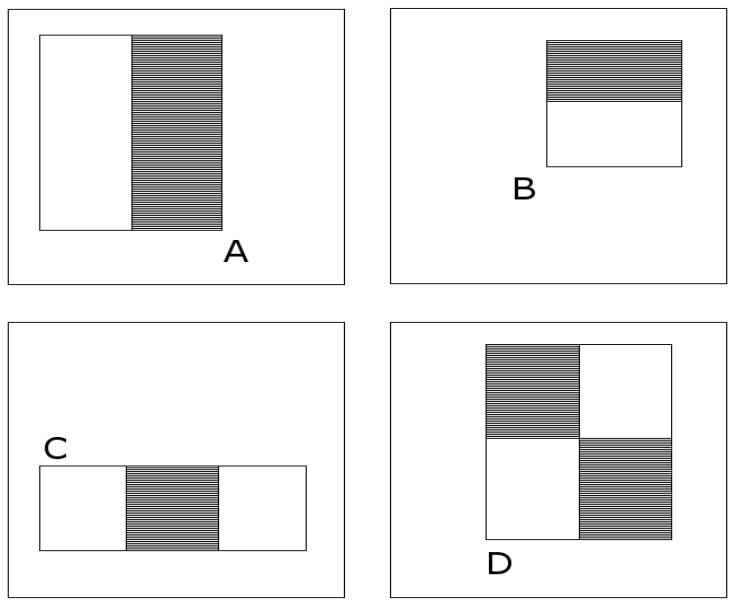

Fig.5: Haar features used in viola-jones algorithm.

For instance, when detecting face, feature (c) is utilized to identify the eye pair and nose.

\subsection{Feature extraction}

Stress is an outcome of negative emotions. A few key activation points were utilized to identify the emotions and classify them. Most of the voluntary muscles of the face are contributes to the detecting algorithm. The viola-jones algorithm was trained to create a detector or handle that can be used to identify these key points. A Histogram Of Gradients (HOG) feature, a technique that counts occurrences of gradient orientation in localized portions of an image, is utilized to train the detector. 


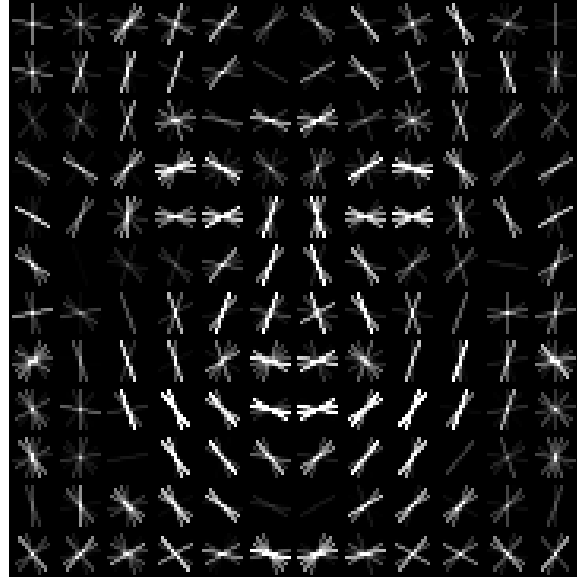

Fig.6: HOG feature of a face.

\subsection{Emotion Recognition}

The emotions are to be extracted from the detected face. The image that is captured from the camera module, contains the facial features. The detected face is pre-processed (i.e.) cropped and resized. The detectors defined prior can be utilized to identify the emotion and sort them. It must be noted that viola-jones algorithm uses adaboost algorithm with cascading classifier, wherein a series of weak classifier's classification with a satisfactory threshold is combined to give an acceptable outcome.

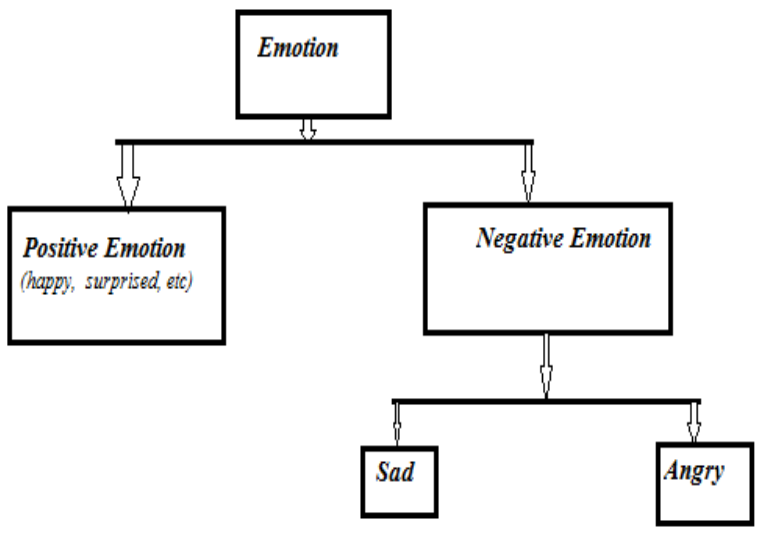

Fig.7: Simple Classification of emotions

\section{Stress Threshold:}

After the emotion is detected, it is plotted against the time axis. Over a period of time it is observed and if it crosses an estimated threshold value, some action is performed.

\subsection{Activity}

A vent to express the stress out would prove to be a wonderful method of getting relieved from stress. Few of the probable activities in case stress is detected are playing music in case where the threshold is acutely high or creating a call setup to any loved ones in case the peak is reached gradually.

\section{Result}

The video from which snapshots were detected has been trained to deal with one face at a time. The algorithm runs with a very low false positive and almost no false negative. The number of false positive can be reduced by augmenting the system with a voice recognition system that implements single word recognition like Alexa, training it to identify negative words and thus improving the overall response of the system.

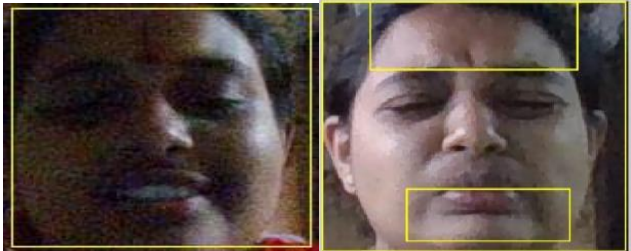

(a)

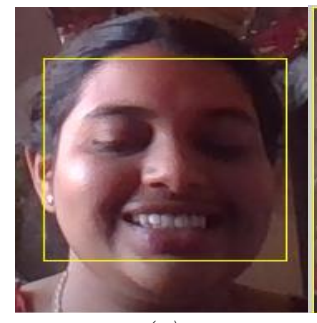

(a) (b)

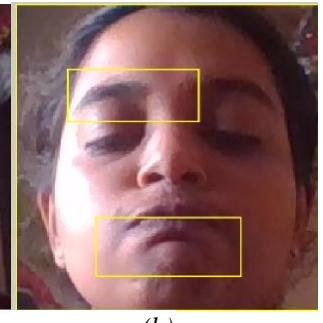

(b)
Fig.8: Sample image from live video. (a) positive emotion (b) negative emotion

The result obtained is plotted with the no of trails in $\mathrm{x}$-axis and the percentage accuracy in the $y$-axis.

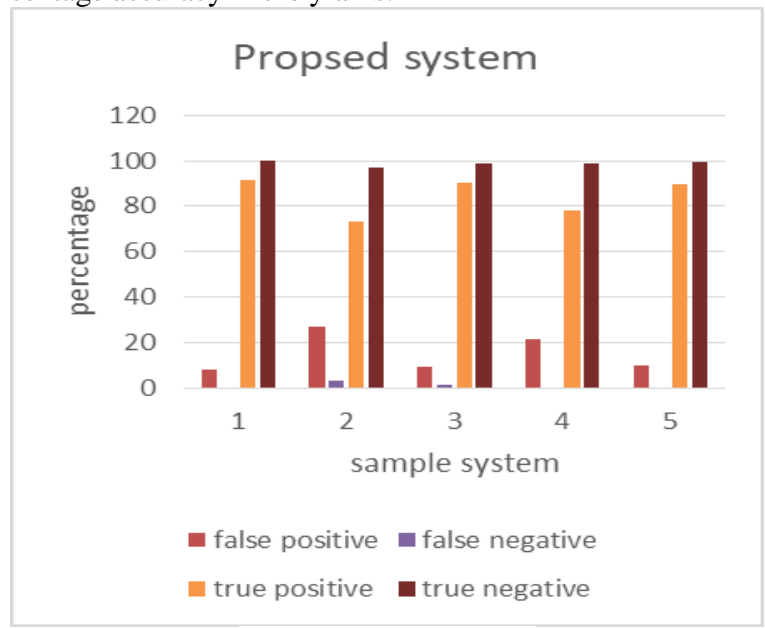

Fig.9: Shows this plotting

\section{Conclusion}

The proposed system detects the face using haar features with viola-jones algorithm, detects and extracts features based on training provided with hog features with viola-jones. This model gives a low false positive rate which can be corrected by augmenting it with other emotion detection technique like word recognition. Future indications include migrating this whole system to a more mobile environment so as to gather more images of the subject, improve the action to be performed choices.

\section{References}

[1] Dr. Margaret Chan, "Preventing suicide a global imperative", World Health Organization, (2014).

[2] Rajiv Radhakrishnan, Chittaranjan, "Suicide: An Indian perspective", Indian Journal of Psychiatry, (2012).

[3] Soman C, Vijayakumar K, Ajayan K, Safraj S, Kutty V, "Suicide in South India: a community-based study in Kerala", Indian J Psychiatry, (2009), Vol.51, pp.261-264.

[4] Deb, Esben Strodl, Jiandong Sun, "Academic Stress, Parental Pressure, Anxiety and Mental Health among Indian High School Students", International Journal of Psychology and Behavioral Science, (2015), Vol.5, Issue.1, pp.26-34.

[5] Vikram Patel, Chinthanie Ramasundarahettige, Lakshmi Vijayakumar, J S Thakur, Vendhan Gajalakshmi, Gopalkrishna Gururaj, Wilson Suraweera, Prabhat Jha, "Suicide mortality in India: a na- 
tionally representative survey", The Lancet, (2012), Vol.379, Issue. 9834, pp.2343-2351.

[6] P. Viola and M. Jones, "Rapid object detection was using a boosted cascade of simple features", CVPR, (2001), pp.511-518.

[7] Damir Filko, Goran Martinovi'c, "Emotion Recognition System by a Neural Network Based Facial Expression Analysis", Automatika, (2013), Vol.54, Issue.2, pp 263-272.

[8] Neha Gupta1 and Navneet Kaur, "Design and Implementation of Emotion Recognition System by Using Matlab", IJERA, (2013), Vol.3, Issue 4, pp.2002-2006.

[9] Seyedehsamaneh Shojaeilangari, Wei-Yun Yau, Karthik Nandakumar, Li Jun, and Eam Khwang Teoh, "Robust Representation and Recognition of Facial Emotions Using Extreme Sparse Learning", IEEE Transactions on Image Processing, (2015), Vol.24, No.7, pp.2140-2153.

[10] Bosker, Bianca, "AFFECTIVA's Emotion Recognition Tech: When Machines Know what you're feeling", (2013).

[11] Vikramjit Mitra1etal, "Cross-Corpus Depression Prediction From Speech", ICASSP, (2015), pp.4769-4773.

[12] Fuji Ren, Xin Kang, and Changqin Quan "Examining Accumulated Emotional Traits in Suicide Blogs with an Emotion Topic Model" IEEE Journal Of Biomedical And Health Informatics, (2016), Vol.20, Issue.5, pp.1348-1351.

[13] Mohammad Soleymani, Sadjad Asghari-Esfeden, Yun Fu, Maja Pantic, "Analysis of EEG Signals and Facial Expressions for Continuous Emotion Detection", IEEE Transactions On Affective Computing, (2016), Vol.7, Issue.1, pp.17-28. 